\title{
Gender role stereotypes, patriarchal attitudes, and cognitive function in the elderly rural Korean population: a cross-sectional study
}

\author{
Hye Rin Choi', Byeonggwan $\mathrm{Ha}^{2}$, Ye Jin Jeon ${ }^{3}$, Yoosik Youm ${ }^{4}$, Hyeon Chang Kim, \\ Sun Jae Jung ${ }^{3,5}$ \\ ${ }^{1}$ Healthcare Data Promotion Division, Bureau of Health Industry, Ministry of Health and Welfare, Sejong, Korea; ${ }^{2}$ Yonsei University College \\ of Medicine, Seoul, Korea; ${ }^{3}$ Department of Public Health, Yonsei University Graduate School, Seoul, Korea; ${ }^{4}$ Department of Sociology, Yonsei \\ University College of Sociology, Seoul, Korea; ${ }^{5}$ Department of Preventive Medicine, Yonsei University College of Medicine, Seoul, Korea
}

OBJECTIVES: We analyzed whether gender role stereotypes (GRS) and patriarchal attitudes are associated with cognitive function in an elderly community.

METHODS: We analyzed data from 580 people enrolled in the Korean Social Life, Health, and Aging Project. The degrees to which respondents held stereotypical beliefs about gender roles and had patriarchal mindsets were measured using a questionnaire. Based on participants' responses, we divided respondents into 2 groups-those with conservative mindsets and those with open mindsets-according to the median score for each of the 2 variables. Cognitive function was assessed using the Mini-Mental State Examination, Korean version (MMSE-K). Cognitive impairment was defined as an MMSE-K score $\leq 21$ points. Multivariable logistic regression was performed, adjusting for gender, age, socio-demographic and lifestyle factors, and social network size. Age and lifestyle factors were stratified.

RESULTS: Compared to those with open mindsets, those with conservative mindsets regarding gender roles and patriarchal norms had adjusted odds ratios of 1.88 (95\% confidence interval [CI], 1.11 to 3.19) and 1.67 (95\% CI, 1.00 to 2.79) for cognitive impairment, respectively. In the stratified analysis, subgroups with younger age and a good lifestyle maintained a protective association with cognitive impairment.

CONCLUSIONS: GRS and a patriarchal mindset were marginally significantly associated with cognitive impairment among women later in life.

KEY WORDS: Gender role, Stereotyping, Cognitive dysfunction, Geriatrics, Public health

\section{Correspondence: Sun Jae Jung}

Department of Preventive Medicine, Yonsei University College of Medicine, 50-1 Yonsei-ro, Seodaemun-gu, Seoul 03722, Korea E-mail: sunjaejung@yuhs.ac

Received: Feb 15, 2021 / Accepted: Apr 7, 2021 / Published: Apr 7, 2021

This article is available from: https://e-epih.org/

(C) This is an open-access article distributed under the terms of the Creative Commons Attribution License (https://creativecommons.org/licenses/by/4.0/), which permits unrestricted use, distribution, and reproduction in any medium, provided the original work is properly cited.

(C) 2021, Korean Society of Epidemiology

\section{INTRODUCTION}

More than 50 million people worldwide have dementia, and that number is estimated to triple by 2050 [1,2]. The rapid growth of the elderly population and changes in familial structure are increasing the burden of dementia [3]. Previous studies investigating gender differences in cognitive function among the elderly attributed these differences to biological factors such as estrogen or apolipoprotein E [4]. However, more recent studies have focused on psychosocial aspects instead, such as education and participation in the labor force [5].

Gender role stereotypes (GRS) refer to the general social expec- 
tations placed on members of particular genders. Their cognitive and motivational function affects how people perceive and engage in the world they live in [6]. The term "patriarchy" is defined in various ways based on its use in diverse academic fields such as sociology, anthropology, and women's studies [7-9]. In our study, we defined "patriarchy" as a social and familial structure in which men hold more power than women, which is reflected in wider society [10]. It has been suggested that patriarchal mindsets have a major influence on individual behavior, from everyday family affairs to major life-changing decisions. Patriarchal status is inherited through the men lineage. Patriarchal norms and GRS affect peoples' lifestyles, and patriarchal beliefs, such as the idea that men should dominate over women, which can influence relationships between people of different genders $[9,11]$. However, patriarchal norms also include concepts regarding inter-generational relationships, such as the dominance of older generations over younger generations, an emphasis on obedience for children, and familial preference for the eldest son [11]. Previous studies have suggested that attitudes toward traditional gender roles or patriarchal norms can constrain or encourage individuals to make certain choices because of their gender, which thereby influence cognitive function later in life [5,12-14]. Unlike GRS, few studies have investigated the impact of patriarchal attitudes on cognitive function, even though such attitudes are an important factor influencing how people act within the circumstances of their lives $[15,16]$. Furthermore, most previous studies were conducted in Western countries, and the association between GRS and cognitive impairment has not been fully investigated in an East Asian context. East Asian culture has relatively pronounced patriarchal qualities and Confucian values, which are associated with a higher prevalence of mental disorders [17]. Korea, as an East Asian country, has historically shown a strong cultural tendency toward stereotypical gender roles and patriarchal norms, with those tendencies being most pronounced among the elderly rural population [18]. Therefore, we aimed to investigate the association of GRS and patriarchal mindsets with cognitive impairment in a rural Korean population.

\section{MATERIALS AND METHODS}

\section{Study population}

The data for this study were collected from the Korean Social Life, Health, and Aging Project (KSHAP) cohort study initiated in 2011. The KSHAP study recruited individuals aged 60 or older, and their spouses, who lived in Township K, Ganghwa Island, Incheon, Korea [19]. With the aid of township officers, after performing a pilot study, a total of 860 people were identified as the target population of KSHAP [20]. To obtain consent and administer questionnaires, we visited participants' homes individually. In total, 814 of the 860 community-dwelling adults (response rate, 94.7\%) participated in the study and completed questionnaire surveys from December 2011 to July 2012 [21]. After the baseline study began, we conducted additional follow-up surveys and health examinations 4 times until 2019. In 2017, we conducted an additional baseline-

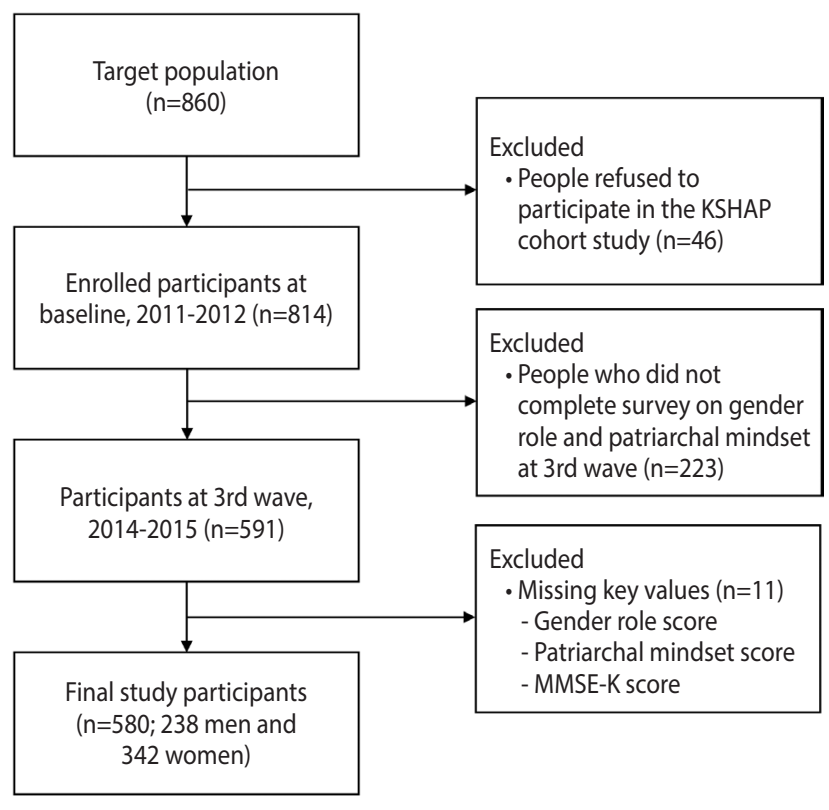

Figure 1. Flow chart of the study population. KSHAP, Korean Social Life, Health, and Aging Project; MMSE-K, Mini-Mental State Examination, Korean version.

only study on 948 participants living in Township L, Ganghwa Island, Incheon, Korea. We collected data on GRS in the same way as in Township K. Since the questionnaires about GRS and patriarchal attitudes were only included in the third wave in Township $\mathrm{K}$, we conducted a cross-sectional analysis using only the thirdwave data $(n=591)$. We also used data on GRS in Township L to replicate the main results in Township K. We excluded 11 participants from the study due to missing key variables such as total scores for views on gender roles $(n=8)$, patriarchal attitudes $(n=1)$, and cognitive function $(n=2)$, leaving 580 people $(238$ men and 342 women) for the final analysis (Figure 1).

\section{Measurements}

Our trained personnel interviewed participants using standardized questionnaire surveys according to a pre-defined protocol. We used the questionnaires to obtain information on socio-demographic characteristics including age, education, economic and marital status, health behaviors, medical history, depressive symptoms, social network size, quality of the spousal relationship, and network constraints of spouse. We measured the quality of the spousal relationship by calculating the frequency of communication and degree of emotional intimacy between spouses. Spouse constraints refer to the high-density networks surrounding a married couple [22]. We calculated network constraints of spouse based on social network information using a network approach, as per previous studies $[23,24]$. Cognitive function was assessed using the Mini-Mental State Examination, Korean version (MMSE-K), which has been widely applied in epidemiological studies [25]. The scores of the MMSE-K range from 0 points to 30 points. Cognitive 
impairment was defined as an MMSE-K score of $\leq 21$ points, based on the cut-off recommended in previous studies [26].

We asked about participants' beliefs regarding gender roles using a questionnaire developed by combining 2 parts of the International Social Survey Programme (ISSP) 2002 Family and Changing Gender Roles III module. The ISSP is an ongoing cross-national collaboration to develop surveys for important social science research [27]. Since this survey has already been used in numerous studies, it has high validation levels, and 1 of its main topics is GRS in the family [28]. Therefore, we used 2 statements from the ISSP questionnaire to measure beliefs regarding stereotypical gender roles in our study population. The statements on GRS were as follows: (1) "Both the man and woman should contribute to household income," and (2) "A man's job is to earn money, a woman's job is to look after the home and family." Each statement had five possible responses: "strongly agree," "agree," "neither agree nor disagree," "disagree," and "strongly disagree." Responses were scored using a corresponding numerical scale ranging from 1 ("strongly agree") to 5 ("strongly disagree") points. Possible total scores ranged from 2 points to 10 points. People with lower scores had a more conservative mindset regarding GRS, while those with higher scores had a more open mindset. Thus, for statistical analysis, we divided participants into 2 groups-conservative mindset and open mindsetaccording to the median score total to analyze beliefs about GRS.

Using a similar method, we conducted another questionnaire to measure patriarchal attitudes using parts from the East Asian Social Survey (EASS) 2006 family module. The EASS is a social survey project and one of the few internationally-coordinated social survey data collection efforts focused on East Asia [29]. The survey examined the traditional patriarchal attitudes of Korean, Chinese, Japanese, and Taiwanese respondents. We selected the following 5 statements from the survey to measure patriarchal attitudes: (1) "the authority of the father in a family should be respected under any circumstances," (2) "children must make efforts to do something that would bring honor to their parents," (3) "the eldest son should inherit a larger share of the property," (4) "a child who has taken good care of parents should inherit a larger share of the property," and (5) "to continue the family line, one must have at least one son." Participants selected 1 of 7 possible responses: "strongly agree," "fairly agree," "somewhat agree," "neither agree nor disagree," "somewhat disagree," "fairly disagree," and "strongly disagree." Responses were measured using a corresponding numerical scale ranging from 1 ("strongly agree") to 7 ("strongly disagree") points. Possible total scores for patriarchal mindset ranged from 5 points to 35 points. As for GRS scores, a lower point total indicated a more conservative patriarchal mindset, and a higher total indicated a more open mindset. For statistical analysis, participants were divided into open mindset and conservative mindset groups according to the median score total for patriarchal attitudes. Using binary variables allowed us to concentrate more on the implications of the results. For the model used to assess the association between GRS, patriarchal attitudes, and cognitive function, we selected covariates based on previous studies, including age [30], education [31], household income [32], hypertension [33], physical activity [34], depressive symptoms [35], social network size [36], smoking status [37], and drinking status [38].

\section{Statistical analysis}

The differences between socio-demographic characteristics according to beliefs regarding GRS and patriarchal attitudes were analyzed using the independent t-test for continuous variables and the chi-square test for categorical variables. Continuous variables that followed a normal distribution were shown as mean and standard deviation, whereas skewed variables were expressed as median and interquartile range. Categorical variables were described as numbers and percentages. We divided views on GRS and patriarchal attitudes into 2 groups-conservative mindset and open mindset-according to the median scores for each. To evaluate the independent associations of views on GRS and patriarchal attitudes with cognitive impairment, we performed multiple logistic regression analyses of men and women, employing unadjusted and fully adjusted models. The full model was adjusted for gender, age, household income, education, hypertension, physical activity, depressive symptoms, social network size, smoking status, and drinking status. Furthermore, we performed subgroup analyses of groups divided by quality of spousal relationship, network constraints of spouse [39], age [30], and lifestyle [37,38], based on the analyses of previous papers. Because GRS and patriarchal attitudes are usually influenced by social relationships and environment, the impacts of age, lifestyle, and social network needed to be investigated to determine whether they interacted with or were effect modifiers for associations between views on GRS, patriarchal attitudes, and cognitive impairment. The quality of spousal relationship and network constraints of spouse were only divided according to their median values for analyzing GRS, and not for patriarchal attitudes, since GRS was based on the spousal relationship. We divided age into 2 groups according to the median age of 73 . We divided frequency of physical activity into 3 groups: less than once per week, 1-4 times per week, and 5 or more times per week. Social network size was classified into 2 groups according to the median level of 3. Depressive symptoms were assessed using the Center for Epidemiologic Studies Depression scale. We used the variables for drinking and smoking status to delineate lifestyle behaviors into 2 groups, namely "good" and "bad." We operationally defined the "good lifestyle" group as consisting of those who reported they were never, or were former but not current, drinkers and smokers. Current drinkers or smokers comprised the "bad lifestyle" group. All analyses were performed using SAS version 9.4 (SAS Institute Inc., Cary, NC, USA). Statistical significance was defined as a 2-sided p-value of less than 0.05 .

\section{Ethics statement}

The Institutional Review Board of Yonsei University (YUIRB2011-012-01) approved the protocol of the KSHAP study. All methods were performed in accordance with relevant guidelines and regulations. Informed consent was obtained from all participants. 


\section{RESULTS}

Table 1 presents the general characteristics of the study population according to beliefs regarding GRS and patriarchal attitudes.
The mean ages were significantly higher for groups with conservative mindsets regarding GRS or patriarchal norms than for openminded groups. The proportion of respondents with an education level lower than elementary school was significantly higher for

Table 1. General characteristics of the study population according to beliefs regarding gender role stereotypes and patriarchal attitudes

\begin{tabular}{|c|c|c|c|c|c|c|c|}
\hline \multirow[b]{2}{*}{ Characteristics } & \multirow{2}{*}{$\begin{array}{c}\text { Total } \\
(n=580)\end{array}$} & \multicolumn{2}{|c|}{ Gender role } & \multirow[b]{2}{*}{$p$-value } & \multicolumn{2}{|c|}{ Patriarchal attitude } & \multirow[b]{2}{*}{$p$-value } \\
\hline & & $\begin{array}{c}\text { Open } \\
(n=256)\end{array}$ & $\begin{array}{c}\text { Conservative } \\
(n=324)\end{array}$ & & $\begin{array}{l}\text { Weak } \\
(n=276)\end{array}$ & $\begin{array}{l}\text { Strong } \\
(n=304)\end{array}$ & \\
\hline Age (yr) & $72.64 \pm 7.01$ & $71.34 \pm 6.94$ & $73.69 \pm 7.04$ & $<0.001$ & $71.54 \pm 7.22$ & $73.57 \pm 6.65$ & $<0.001$ \\
\hline Gender, men & $238(41.0)$ & $100(39.1)$ & $138(42.6)$ & 0.391 & 115 (41.7) & $123(40.5)$ & 0.768 \\
\hline \multicolumn{8}{|l|}{ Household income ( $\left.10^{4} \mathrm{KRW}\right)$} \\
\hline$<1,000$ & $314(54.1)$ & $121(47.3)$ & $193(59.6)$ & 0.002 & $141(51.1)$ & $173(56.9)$ & 0.538 \\
\hline$<2,000$ & $124(21.4)$ & $66(25.8)$ & $58(17.9)$ & & $66(23.9)$ & $58(19.1)$ & \\
\hline$<3,000$ & $53(9.1)$ & $25(9.8)$ & $28(8.6)$ & & $26(9.4)$ & $27(8.9)$ & \\
\hline$\geq 3,000$ & $46(7.9)$ & $29(11.3)$ & $17(5.3)$ & & $24(8.7)$ & $22(7.2)$ & \\
\hline \multicolumn{8}{|l|}{ Education } \\
\hline Less than elementary school & $197(34.0)$ & 68 (26.6) & $129(39.8)$ & 0.001 & $83(30.1)$ & $114(37.5)$ & 0.012 \\
\hline Elementary school & $224(38.6)$ & $102(39.8)$ & $122(37.7)$ & & $101(36.6)$ & $123(40.5)$ & \\
\hline Middle school & 79 (13.6) & $42(16.4)$ & 37 (11.4) & & $41(14.9)$ & $38(12.5)$ & \\
\hline High school & $55(9.5)$ & $26(10.2)$ & $29(9.0)$ & & $33(12.0)$ & $22(7.2)$ & \\
\hline$\geq$ College & $25(4.3)$ & $18(7.0)$ & $7(2.2)$ & & $18(6.5)$ & $7(2.3)$ & \\
\hline Hypertension & $284(49.3)$ & $117(46.1)$ & $167(51.9)$ & 0.167 & $140(51.1)$ & $144(47.7)$ & 0.413 \\
\hline \multicolumn{8}{|l|}{ Physical activity (times/wk) } \\
\hline$<1$ & $280(48.3)$ & $110(43.0)$ & $170(52.5)$ & 0.080 & $133(48.2)$ & $147(48.3)$ & 0.248 \\
\hline $1-4$ & $66(11.4)$ & $32(12.5)$ & $34(10.5)$ & & $35(12.7)$ & $31(10.2)$ & \\
\hline$\geq 5$ & $71(12.2)$ & $75(29.3)$ & $88(27.2)$ & & $39(14.1)$ & $32(10.5)$ & \\
\hline \multicolumn{8}{|l|}{ Social network size } \\
\hline$\leq 3$ & $353(61.3)$ & $151(59.7)$ & $202(62.5)$ & 0.485 & $168(61.5)$ & $185(61.1)$ & 0.905 \\
\hline$>3$ & $223(38.7)$ & $102(40.3)$ & $121(37.5)$ & & $105(38.5)$ & $118(38.9)$ & \\
\hline \multicolumn{8}{|c|}{ Quality of relationship with spouse } \\
\hline Weak & $294(50.7)$ & $125(48.8)$ & $169(52.2)$ & 0.425 & $146(52.9)$ & $148(48.7)$ & 0.311 \\
\hline Strong & $286(49.3)$ & $131(51.2)$ & $155(47.8)$ & & $130(47.1)$ & $156(51.3)$ & \\
\hline \multicolumn{8}{|l|}{ Spouse constraints } \\
\hline Weak & $363(62.6)$ & $160(62.5)$ & $203(62.7)$ & 0.970 & $183(66.3)$ & $180(59.2)$ & 0.078 \\
\hline Strong & $217(37.4)$ & $96(27.5)$ & $121(38.4)$ & & $93(33.7)$ & $124(40.8)$ & \\
\hline \multicolumn{8}{|l|}{ Smoking status } \\
\hline Former/non-smokers & $522(90.0)$ & $230(89.8)$ & $292(90.1)$ & 0.911 & $243(88.0)$ & $279(91.8)$ & 0.134 \\
\hline Current smokers & $58(10.0)$ & $26(10.2)$ & $32(9.9)$ & & $33(12.0)$ & $25(8.2)$ & \\
\hline \multicolumn{8}{|l|}{ Drinking status } \\
\hline Former/non-drinkers & $469(80.9)$ & $213(83.2)$ & $256(79.0)$ & 0.203 & $218(79.0)$ & $251(82.6)$ & 0.274 \\
\hline Current drinkers & $111(19.1)$ & $43(16.8)$ & $68(21.0)$ & & $58(21.0)$ & $53(17.4)$ & \\
\hline Gender role score & $6.77 \pm 1.72$ & $8.33 \pm 1.13$ & $5.54 \pm 0.97$ & $<0.001$ & $7.13 \pm 1.76$ & $6.45 \pm 1.63$ & $<0.001$ \\
\hline Patriarchal mindset score & $13.74 \pm 5.87$ & $15.22 \pm 5.90$ & $12.58 \pm 5.50$ & 0.002 & $18.77 \pm 4.02$ & $9.18 \pm 2.69$ & $<0.001$ \\
\hline CES-D & $4[1,10]$ & $3[1,9]$ & $5[2,11]$ & $<0.001$ & $4[1,11]$ & $4[1,10]$ & 0.428 \\
\hline MMSE-K & $25[22,27]$ & $26[23,28]$ & $24[21,27]$ & $<0.001$ & $26[22,28]$ & $24[21,27]$ & 0.024 \\
\hline \multicolumn{8}{|l|}{ MMSE-K, category } \\
\hline Normal cognitive function ${ }^{1}$ & $437(75.3)$ & $216(84.4)$ & $221(68.2)$ & $<0.001$ & $221(80.1)$ & $216(71.1)$ & 0.012 \\
\hline Cognitive decline ${ }^{2}$ & $143(24.7)$ & $40(15.6)$ & $103(31.8)$ & & $55(19.9)$ & $88(28.9)$ & \\
\hline
\end{tabular}

Values are presented as mean \pm standard deviation, median [interquartile range], or number (\%).

KRW, Korean won; CES-D, Center for Epidemiology Studies Depression scale; MMSE-K, Mini-Mental Status Examination, Korean version. ${ }^{1}$ Normal cognitive function was defined as MMSE-K $>21$ points.

${ }^{2}$ Cognitive decline was defined as MMSE- $K \leq 21$ points. 
conservative groups than for open-minded groups. The mean MMSE-K scores were significantly lower for conservative groups compared to the open-minded groups. We also provide data on differences in general characteristics by gender in Supplementary Material 1 and differences in general characteristics by cognitive status in Supplementary Material 2.

Figure 2 shows the association of beliefs regarding GRS and patriarchal attitudes with cognitive impairment in total, in men only, and in women only. Of the total number of participants, the unadjusted odds ratio (OR) of a conservative mindset toward gender roles for having cognitive impairment was 2.52 (95\% confidence interval [CI], 1.67 to 3.79) compared to an open mindset. After adjusting for gender, age, household income, education, hypertension, physical activity, depressive symptoms, social network size, smoking status, and drinking status, the adjusted OR for cognitive impairment was 1.88 (95\% CI, 1.11 to 3.19) in the group with a conservative mindset toward GRS compared to the open-minded group. Furthermore, the adjusted ORs for cognitive impairment were 2.16 (95\% CI, 0.66 to 7.05 ) and 1.80 (95\% CI, 0.98 to 3.32) for men and women, respectively, in the group with a conservative mindset toward GRS. Moreover, there was no significant interaction between beliefs regarding GRS and gender $(p=0.696)$. Regarding patriarchal attitudes, among all participants, the unadjusted OR for cognitive impairment was 1.64 (95\% CI, 1.11 to 2.41 ) for people with a conservative mindset toward patriarchy compared to the group with an open mindset. After adjusting for po- tential confounders, the ORs for cognitive impairment were 1.27 ( $95 \%$ CI, 0.43 to 3.71) and 1.82 (95\% CI, 0.99 to 3.33) in men and women, respectively, in the group with a conservative mindset toward patriarchy. The interaction between patriarchal attitudes and gender was not significant $(\mathrm{p}=0.529)$. To replicate these results, we performed another robust test using an independent sample consisting of participants living in Township L. As shown in Supplementary Material 3, we found that the significant association between beliefs regarding GRS and cognitive impairment was consistent with our main results for participants living in Township K. To support the main results in Figure 2, we also examined the associations of beliefs regarding GRS and patriarchal attitudes with continuous MMSE-K scores using linear regression analysis, shown in Supplementary Materials 3 and 4, respectively. People with conservative beliefs regarding GRS were more likely to have low MMSE-K scores than those in the open-minded group. Similarly, those with a conservative mindset toward patriarchal norms were also more likely to have low MMSE-K scores, but the results were not statistically significant.

Figure 3 shows the association of beliefs regarding GRS and patriarchal attitudes with cognitive impairment from the subgroup analysis of age and lifestyle behaviors. In addition, we analyzed the associations between beliefs regarding GRS and cognitive impairment in subgroups defined according to the quality of spousal relationship and network constraints of spouse, since the quality of spousal relationship was only related to GRS, and not patriar-

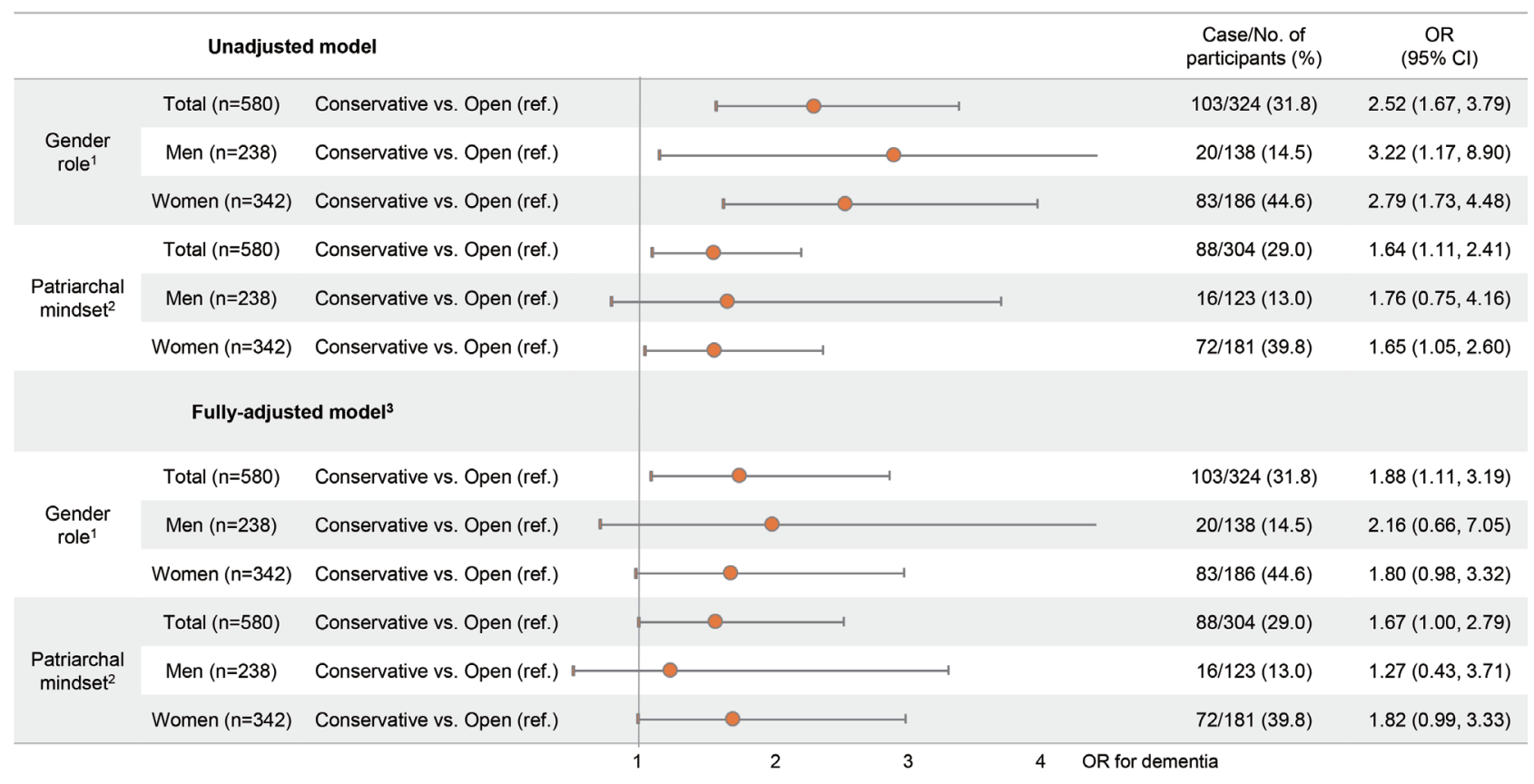

Figure 2. Odds ratios (ORs) for cognitive impairment among the total study population, men only, and women only in unadjusted and fullyadjusted models. $\mathrm{Cl}$, confidence interval; ref., reference. 'People with open attitude to gender role stereotype were defined as those with over the median score, 6 points, of gender role questionnaires. ${ }^{2}$ People with open attitude to patriarchal mindset were defined as those with over the median score, 13 points, of patriarchy questionnaires. ${ }^{3}$ Adjusted for gender, age, household income, education, hypertension, physical activity, depressive symptoms, social network size, smoking and drinking status. 


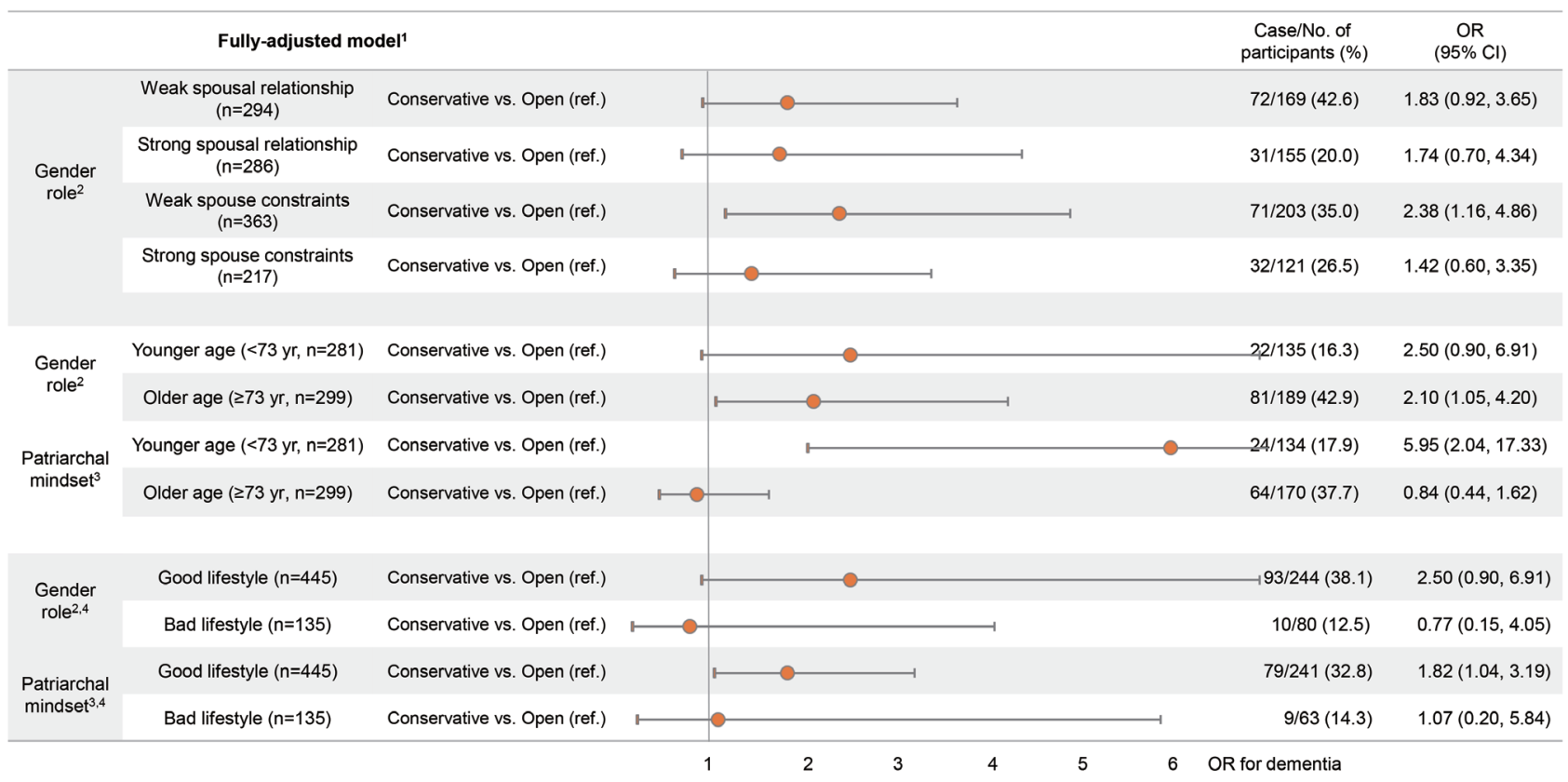

Figure 3. Odds ratios (ORs) for cognitive impairment among groups stratified by quality of spousal relationship, spouse constraints, age, and lifestyle in the fully-adjusted model. $\mathrm{Cl}$, confidence interval; ref., reference. ${ }^{1}$ Adjusted for gender, age, household income, education, hypertension, physical activity, depressive symptoms, social network size, smoking and drinking status. ${ }^{2}$ People with open mind to gender role were defined as those with over the median score, 6 points, of gender role questionnaires. ${ }^{3}$ People with open mind to patriarchal mindset were defined as those with over the median score, 13 points, of patriarchy questionnaires. ${ }^{4} \mathrm{Good}$ lifestyle group consists of never or former drinkers and smokers; bad lifestyle group consists of current drinkers or current smokers.

chal attitudes. For the subgroups with weak spousal relationships and spousal constraints, the fully adjusted ORs for cognitive impairment were 1.83 (95\% CI, 0.92 to 3.65 ) and 2.38 (95\% CI, 1.16 to 4.86$)$, respectively, for those with conservative mindsets regarding GRS compared to those with open mindsets. The older subgroup ( $\geq 73$ years old) with a conservative mindset toward GRS had a significantly higher (OR, 2.10; 95\% CI, 1.05 to 4.20 ) for cognitive impairment than older people with open mindsets did. For those with a good lifestyle, the adjusted ORs for cognitive impairment were 2.50 (95\% CI, 0.90 to 6.91 ) and 1.82 (95\% CI, 1.04 to 3.19) for groups with conservative mindsets toward GRS and patriarchal norms, respectively, compared to groups with open mindsets. The results for the association between patriarchal attitudes and cognitive impairment in the younger group showed that the adjusted OR for cognitive impairment was significantly higher for those with conservative mindsets toward patriarchal norms. In addition, there was a significant interaction between patriarchal attitudes and age $(\mathrm{p}=0.006)$.

\section{DISCUSSION}

We observed marginally significant associations of beliefs regarding GRS and patriarchal attitudes with cognitive impairment in women after adjusting for potential confounders. Additionally, we found a significant positive association between beliefs regarding GRS and cognitive impairment among groups with weak net- work constraints of spouse and a high age. After stratifying by age, people with conservative mindsets toward patriarchal norms who were relatively younger had a significantly higher tendency for having cognitive impairment. Thus, we suggest that age is an effect modifier for the association between patriarchal attitudes and cognitive function. Additionally, there was a significant positive relationship between patriarchal attitudes and cognitive impairment among the group with a good lifestyle.

Whether norms affect cognitive function has been studied with more focus on attitudes toward gender roles than on patriarchal norms. Several previous studies support our findings [40-44]. According to a previous study from the United States [40], men with strong conservative views regarding gender roles and a high degree of qualities aligned with traditional masculinity are significantly more likely to commit suicide than those who do not have such views or qualities. Another cross-sectional study investigated the relationship between patriarchal attitudes and men's health by comparing women's homicide rates to men's mortality rates [41]. They found that nations with high-levels of patriarchal attitudes were significantly associated with higher mortality rates for men. The results suggest that oppression and exploitation harm not only the oppressed, but also the oppressors. We can also infer that highly gender-typed people are more likely to have mental disorders, such as depression and cognitive impairment. In our study, men with conservative beliefs regarding GRS had a much higher OR for cognitive impairment than women with conservative be- 
liefs regarding GRS. Unlike conservative women, men with conservative attitudes toward GRS are more likely to have qualities aligned with traditional masculinity, such as competitiveness, emotional restriction, and aggression [45]. As a result, men with conservative beliefs regarding GRS may suffer negative effects from both GRS and a high degree of traditional masculinity. This can lead to a higher risk of cognitive impairment than women. An earlier case-control study investigated the effects of GRS on cognitive performance [42]. This study found that men with conservative attitudes toward gender stereotypes had significantly lower verbal fluency abilities than the control group, and even conservative women. From a young age, men and women are conditioned with gender stereotypes regarding what subjects they should excel in. For example, while boys are encouraged to excel at math, girls may be encouraged to excel at language skills. This gender stereotype could result in stress and affect mental health throughout men's and women's lives. Thus, the study suggested that their results may have been due to the threat posed by stereotypes on men's verbal abilities. For another previous study, wholebrain functional magnetic resonance imaging was performed on 3 groups of women-positive-stereotype, negative-stereotype, and control groups [43]. Before undertaking the imagined self-rotation task, the positive-stereotype group was informed that women perform the task better than men do. The negative-stereotype group received the opposite message, and the control group was provided with neutral information. The results demonstrated that the positive-stereotype group made significantly fewer errors in performance than the control group, while the negative-stereotype group made significantly more errors than the control group. The results showed that gender stereotypes could substantially affect women's cognitive performance. This study supports the findings of other studies that stereotypes could have a negative effect on mental health and cognitive function. A possible explanation based on neuroplasticity theory is that having such stereotypes decreases mental flexibility, resulting in impairments to general cognitive function. The importance of "openness" in learning has been well demonstrated in many studies on academic performance-personality relationships because of its correlation with positive learning approaches, learning motivation, and critical thinking [46-48]. Greater belief in stereotypical gender roles or patriarchal attitudes may indicate less openness, since new information can be overshadowed by stereotypical thinking for those with a strong belief in stereotypes [6]. For example, they may discount accomplishments that are inconsistent with stereotypical expectations [49]. As a result, people who tend to process only stereotypical information may become more vulnerable to cognitive impairment. Moreover, conservative attitudes toward GRS may prevent women from being educated or having an occupational role outside of performing housework, which may cumulatively affect their cognitive function [5].

Our study yielded several interesting findings. First, the group with weaker network constraints of spouse had a significant positive association between GRS and cognitive impairment. Stronger spouse constraints may be an obstacle that prevents a person from having an open mind to non-stereotypical gender roles. For example, a person with strong spouse constraints and their partner may be surrounded by a high-density social network. In such circumstances, even though one may not have a strong belief in GRS, one's freedom to live in a way that goes against social stereotypes may be hindered. Secondly, GRS had a significant positive association with cognitive impairment in the older group, while patriarchal attitudes had a significant positive association with cognitive impairment in the younger group. This suggests that GRS and patriarchal attitudes mainly affect different age groups. This may be due to changes in spousal and familial relationships over time. Lastly, the model tested according to lifestyle suggests that bad lifestyles involving high levels of alcohol consumption and smoking may hinder potential cognitive impairment-preventive mechanisms.

This study has several strengths. First, we administered questionnaires by visiting participants' homes individually. We obtained detailed and precise information on an individual level. Therefore, we could control for various potential confounders such as gender, age, household income, education, physical activity, depressive symptoms, social network size, smoking status, and drinking status. Second, our sample has unique characteristics. The study population consisted of people from a rural area, all of a single ethnic background, who formed a closed community within which most residents were born and raised, meaning that the homogeneity of the baseline population could be assumed. Therefore, our findings are unlikely to have been affected by various confounders such as residential area, ethnicity, or environmental factors.

Nonetheless, there are some limitations. First, since this was a cross-sectional study, we only determined the association of GRS and patriarchal attitudes with cognitive impairment, and we cannot suggest a causal relationship. Furthermore, among the group with cognitive impairment, around $76.2 \%$ did not have access to, and thus did not have the option to attend, elementary school. Based on this, we could consider the possibility of reverse causality-that is, participants' low education level might affect their consistent conservative attitude regarding gender roles and patriarchal norms. Regardless, the significant association of GRS and patriarchal attitudes with cognitive impairment is an important finding in terms of the suggested effect gender equality could have on the prevention of cognitive impairment among the elderly. Second, we only assessed cognitive impairment using the MMSE$\mathrm{K}$ test. However, the MMSE-K has been widely used as a diagnostic tool for assessing cognitive function in clinical practice and research. Furthermore, the MMSE-K has been proven to detect cognitive impairment well with high reliability and accuracy in actual public health settings. Because of the MMSE-K's low cost and ease of use, it is recommended as the first step for measuring cognitive function and assessing cognitive impairment [50]. Third, we analyzed a study population from a single rural community. Even though we generated consistent results after replicating the analysis using data from another township, Township K and Town- 
ship L were located on the same island. Moreover, the study participants were isolated on an island and were mainly engaged in farming and fishing. Their social communities may be more constrained than those of urban dwellers, and their lifestyles are also different from people living in urban areas. Thus, it might be difficult to generalize our results to other elderly populations. Finally, we excluded people diagnosed with severe cognitive impairment from the KSHAP survey, which may introduce selection bias. However, we measured cognitive function as a continuous variable using MMSE-K scores. Therefore, we could compare the degree of belief in stereotypical gender roles and patriarchal attitudes between people with lower and higher MMSE-K scores.

Our cross-sectional findings suggest that GRS and patriarchal attitudes may be independently associated with cognitive impairment in women later in life. We also found a significant association between patriarchal attitudes and cognitive impairment only in relatively younger people. Based on our results, we suggest that the value of gender equality and a more open mindset toward family members' roles should be emphasized at the community level. Education should be provided to foster a more equal and less hierarchical culture for elderly communities. This study also suggests that the need to eliminate stereotypes is as important for the elderly population as it is for other age groups. Recognizing the implicit effects of stereotypes will lead people to have a more open-minded attitude toward gender roles and patriarchal norms and improve cognitive function in the elderly population.

In conclusion, our findings show a significant association of GRS and patriarchal attitudes with cognitive impairment; this is a novel finding in Asian countries with relatively conservative cultures. Studies on cognitive impairment require a multi-pronged approach on an individual, biological level as well as on a populational, psychosocial level. Therefore, this study is meaningful, as it confirmed a significant association reflecting East Asian culture and emotional customs. Further longitudinal studies are required to refine the mechanism and determine causality between stereotypical thinking and cognitive function.

\section{SUPPLEMENTARY MATERIALS}

Supplementary materials are available at https://www.e-epih.org/.

\section{CONFLICT OF INTEREST}

The authors have no conflicts of interest to declare for this study.

\section{FUNDING}

This study was supported by a faculty research grant of Yonsei University College of Medicine for 2019 (6-2019-0114) and Basic Science Research Program through the National Research Foundation of Korea (NRF) funded by the Ministry of Science and ICT (NRF-2020R1C1C1003502).

\section{ACKNOWLEDGEMENTS}

None.

\section{AUTHOR CONTRIBUTIONS}

Conceptualization: SJJ. Data curation: YY, SJJ, HCK. Formal analysis: HRC. Funding acquisition: SJJ, YY. Methodology: HRC. Writing - original draft: BH, HRC. Writing - review \& editing: SJJ, BH, HRC, YY, HCK, YJJ.

\section{ORCID}

Hye Rin Choi: https://orcid.org/0000-0002-4373-2581; Byeonggwan Ha: https://orcid.org/0000-0002-2420-4740; Ye Jin Jeon: https://orcid.org/0000-0002-3780-5668; Yoosik Youm: https://orcid. org/0000-0003-3822-5777; Hyeon Chang Kim: https://orcid.org/ 0000-0001-7867-1240; Sun Jae Jung: https://orcid.org/0000-00025194-7339

\section{REFERENCES}

1. Prince M, Guerchet M, Prina M; Alzheimer's Disease International. Policy brief for heads of government: the global impact of dementia 2013-2050; 2013 [cited 2021 Feb 1]. Available from: https://www.alzint.org/u/2020/08/GlobalImpactDementia2013.pdf.

2. Prince $M$, Guerchet $M$, Prina $M$. The epidemiology and impact of dementia: current state and future trends; 2015 [cited $2021 \mathrm{Feb}$ 1]. Available from: https://www.who.int/mental_health/neurology/dementia/dementia_thematicbrief_epidemiology.pdf.

3. Kim YJ, Han JW, So YS, Seo JY, Kim KY, Kim KW. Prevalence and trends of dementia in Korea: a systematic review and metaanalysis. J Korean Med Sci 2014;29:903-912.

4. Rocca WA, Mielke MM, Vemuri P, Miller VM. Sex and gender differences in the causes of dementia: a narrative review. Maturitas 2014;79:196-201.

5. Bonsang E, Skirbekk V, Staudinger UM. As you sow, so shall you reap: gender-role attitudes and late-life cognition. Psychol Sci 2017; 28:1201-1213.

6. Ellemers N. Gender stereotypes. Annu Rev Psychol 2018;69:275298.

7. Ruggles S. The future of historical family demography. Annu Rev Sociol 2012;38:423-441.

8. Smuts B. The evolutionary origins of patriarchy. Hum Nat 1995; 6:1-32.

9. Kandiyoti D. Bargaining with patriarchy. Gend Soc 1998;2:274290.

10. Harrington A, Marshall BL, Müller HP. Encyclopedia of social theory. London: Routledge; 2006, p. 420-422.

11. Gruber S, Szoltysek M. The patriarchy index: a comparative study of power relations across historical Europe. Hist Fam 2016;21: 133-174

12. Reilly D. Gender, culture, and sex-typed cognitive abilities. PLoS 
One 2012; 7:e39904.

13. Deary IJ, Whiteman MC, Starr JM, Whalley LJ, Fox HC. The impact of childhood intelligence on later life: following up the Scottish mental surveys of 1932 and 1947. J Pers Soc Psychol 2004;86: 130-147.

14. Lei X, Smith JP, Sun X, Zhao Y. Gender differences in cognition in China and reasons for change over time: evidence from CHARLS. J Econ Ageing 2014;4:46-55.

15. Coetzee D. South African education and the ideology of patriarchy. S Afr J Educ 2001;21:300-304.

16. Kambarami M. Femininity, sexuality and culture: patriarchy and female subordination in Zimbabwe; 2006 [cited 2021 Feb 1]. Available from: http://www.arsrc.org/downloads/uhsss/kmabarami.pdf.

17. Niaz U, Hassan S. Culture and mental health of women in SouthEast Asia. World Psychiatry 2006;5:118-120.

18. Jeon GS, Cho SI, Choi K, Jang KS. Gender differences in the prevalence and correlates of elder abuse in a community-dwelling older population in Korea. Int J Environ Res Public Health 2019;16: 100.

19. Youm Y, Laumann EO, Ferraro KF, Waite LJ, Kim HC, Park YR, et al. Social network properties and self-rated health in later life: comparisons from the Korean social life, health, and aging project and the national social life, health and aging project. BMC Geriatr 2014;14:102.

20. Lee JM, Lee WJ, Kim HC, Choi W, Lee J, Sung K, et al. The Korean social life, health and aging project-health examination cohort. Epidemiol Health 2014;36:e2014003.

21. Baek J, Hur NW, Kim HC, Youm Y. Sex-specific effects of social networks on the prevalence, awareness, and control of hypertension among older Korean adults. J Geriatr Cardiol 2016;13:580586.

22. Burt RS, Kilduff M, Tasselli S. Social network analysis: foundations and frontiers on advantage. Annu Rev Psychol 2013;64:527-547.

23. Youm Y, Laumann EO. The effect of structural embeddedness on the division of household labor: a game-theoretic model using a network approach. Ration Soc 2003;15:243-280.

24. Cornwell B, Laumann EO. Network position and sexual dysfunction: implications of partner betweenness for men. AJS 2011;117: 172-208.

25. Folstein MF, Folstein SE, McHugh PR. "Mini-mental state". A practical method for grading the cognitive state of patients for the clinician. J Psychiatr Res 1975;12:189-198.

26. Kim JM, Shin IS, Yoon JS, Lee HY. Comparison of diagnostic validities between MMSE-K and K-MMSE for screening of dementia. J Korean Neuropsychiatr Assoc 2003;42:124-130 (Korean).

27. International Social Survey Programme (ISSP). ISSP 2012 - "family and changing gender roles IV" - ZA No. 5900; 2014 [cited 2021 Feb 1]. Available from: https://www.gesis.org/en/issp/modules/ issp-modules-by-topic/family-and-changing-gender-roles/2012.

28. Walter JG. The adequacy of measures of gender roles attitudes: a review of current measures in omnibus surveys. Qual Quant 2018; 52:829-848.
29. Kim SW, Chang YH, Iwai N, Li L; Inter-University Consortium for Political and Social Research. East Asian Social Survey (EASS), cross-national survey data sets: families in East Asia, 2006 (ICPSR 34606) [cited $2021 \mathrm{Feb} 1$ ]. Available from: http://b2find.eudat. eu/dataset/066ccc0e-e921-5e73-ac9f-ba2194227cb8.

30. Savva GM, Wharton SB, Ince PG, Forster G, Matthews FE, Brayne C. Age, neuropathology, and dementia. N Engl J Med 2009;360: 2302-2309.

31. Sharp ES, Gatz M. Relationship between education and dementia: an updated systematic review. Alzheimer Dis Assoc Disord 2011; 25:289-304.

32. Anttila T, Helkala EL, Kivipelto M, Hallikainen M, Alhainen K, Heinonen $\mathrm{H}$, et al. Midlife income, occupation, APOE status, and dementia: a population-based study. Neurology 2002;59:887-893.

33. Breteler MM, Bots ML, Ott A, Hofman A. Risk factors for vascular disease and dementia. Haemostasis 1998;28:167-173.

34. Larson EB, Wang L, Bowen JD, McCormick WC, Teri L, Crane P, et al. Exercise is associated with reduced risk for incident dementia among persons 65 years of age and older. Ann Intern Med 2006;144:73-81.

35. Potter GG, Steffens DC. Contribution of depression to cognitive impairment and dementia in older adults. Neurologist 2007;13: 105-117.

36. Crooks VC, Lubben J, Petitti DB, Little D, Chiu V. Social network, cognitive function, and dementia incidence among elderly women. Am J Public Health 2008;98:1221-1227.

37. Peters R, Poulter R, Warner J, Beckett N, Burch L, Bulpitt C. Smoking, dementia and cognitive decline in the elderly, a systematic review. BMC Geriatr 2008;8:36.

38. Anttila T, Helkala EL, Viitanen M, Kåreholt I, Fratiglioni L, Winblad B, et al. Alcohol drinking in middle age and subsequent risk of mild cognitive impairment and dementia in old age: a prospective population based study. BMJ 2004;329:539.

39. Windsor TD, Gerstorf D, Pearson E, Ryan LH, Anstey KJ. Positive and negative social exchanges and cognitive aging in young-old adults: differential associations across family, friend, and spouse domains. Psychol Aging 2014;29:28-43.

40. Coleman D, Feigelman W, Rosen Z. Association of high traditional masculinity and risk of suicide death: secondary analysis of the Add Health Study. JAMA Psychiatry 2020;77:435-437.

41. Stanistreet D, Bambra C, Scott-Samuel A. Is patriarchy the source of men's higher mortality? J Epidemiol Community Health 2005; 59:873-876.

42. Hirnstein M, Coloma Andrews L, Hausmann M. Gender-stereotyping and cognitive sex differences in mixed- and same-sex groups. Arch Sex Behav 2014;43:1663-1673.

43. Wraga M, Helt M, Jacobs E, Sullivan K. Neural basis of stereotypeinduced shifts in women's mental rotation performance. Soc Cogn Affect Neurosci 2007;2:12-19.

44. Moè A. Gender difference does not mean genetic difference: externalizing improves performance in mental rotation. Learn Individ Differ 2012;22:20-24.

45. Coleman D. Traditional masculinity as a risk factor for suicidal 
ideation: cross-sectional and prospective evidence from a study of young adults. Arch Suicide Res 2015;19:366-384.

46. Poropat AE. A meta-analysis of the five-factor model of personality and academic performance. Psychol Bull 2009;135:322-338.

47. Tempelaar DT, Gijselaers WH, van der Loeff SS, Nijhuis JF. A structural equation model analyzing the relationship of student achievement motivations and personality factors in a range of academic subject-matter areas. Contemp Educ Psychol 2007;32: 105-131.
48. Bidjerano T, Dai DY. The relationship between the big-five model of personality and self-regulated learning strategies. Learn Individ Differ 2007;17:69-81.

49. Banaji MR, Greenwald AG. Implicit gender stereotyping in judgments of fame. J Pers Soc Psychol 1995;68:181-198.

50. Pezzotti P, Scalmana S, Mastromattei A, Di Lallo D; Progetto Alzheimer Working Group. The accuracy of the MMSE in detecting cognitive impairment when administered by general practitioners: a prospective observational study. BMC Fam Pract 2008;9:29. 